\title{
Further evidence for the effect of passive smoking on neonates
}

\author{
M.J. Campbell, J. Lewry and M. Wailoo* \\ Medical Statistics and Computing, South Academic Block, Southampton General Hospital, Southampton \\ SO9 $4 X Y, U K$.
}

\begin{abstract}
Summary: From 518 couples with a 1 month old baby, information was obtained on the couple's smoking habit, social class, age of mother, parity, alcohol consumption during pregnancy and respiratory symptoms of the baby. Allowing for these factors, a multivariate analysis revealed that, even allowing for mother's smoking habit, babies whose father smoked were lighter at birth by an average of $113 \mathrm{~g}(95 \% \mathrm{CI} 8-216 \mathrm{~g})$ and this effect was unchanged at 1 month $(112 \mathrm{~g}, 95 \% \mathrm{CI}$ $224 \mathrm{~g}$ ). Babies whose mothers smoked were twice as likely to have a cough (Relative Risk $=2.0,95 \%$ CI 1.05-3.68), than those whose mothers did not, but we failed to find a significant association with the father's smoking habit.
\end{abstract}

\section{Introduction}

Recent research has demonstrated the effect of passive smoking on birthweight, ${ }^{1,2}$ although the size of the effect has been disputed, ${ }^{3}$ and on cough in older children. ${ }^{4,5}$ We provide further evidence for the effect of smoking by both parents on birthweight, and the effect of mother's smoking on cough in neonates.

\section{Subjects and methods}

From all babies born in Southampton in 1 year an approximate 1:10 random sample of the singleton, Caucasian babies was taken, and the mothers interviewed 1 month after the birth by health visitors (mean age 32 days, range 20-60 days). Over $90 \%$ of the babies were born at the Princess Anne Maternity Hospital, the remainder at small units throughout the district. The following information was obtained from the parents: age of mother, current smoking habit by either parent, alcohol consumption of mother during pregnancy and of father. The latter was classified as frequency of consumption per week, and also the mother was asked whether during her pregnancy she had changed her alcohol consumption. Information about the baby included weight, type of feed, whether or not the baby was suffering from, or had

Correspondence: M.J. Campbell, B.A., Ph.D.

*Present Address: Department of Child Health, Clinical Sciences Building, Leicester Royal Infirmary, Leicester LE1 5WW, UK

Accepted: 2 March 1988 suffered from snuffles/runny nose or a cough. Each baby was weighed by the midwife at delivery. At 1 month, the babies were weighed by the healthe visitor, the instructions being to weigh the babye naked. Gross discrepancies between the birthweighe and the weight at 1 month were investigated and corrected. Social class of mother was assigned using the Registrar General's classification of occupations. Women whose husbands are unemployed or in the armed forces were grouped separately. Birthweight was obtained from the hospital records. Weight gain of baby was defined as increase in weight from birth divided by age in days. Birthweight and weight gain were analysed by multiple linear regression to allow for confounding factors, and symptoms by multiple logistic regression.

\section{Results}

A total of 549 mothers was interviewed, of whom 518 gave usable information on both their own and their husbands' smoking habits. The mean age of the mothers was 27 years (range 16-45), 25\% were smokers, $16 \%$ were in social classes 1 and 2 , and $69 \%$ reported consuming alcohol during pregnancy. Mean birthweight classified by parental smoking habits is shown in Table I. A multiple regression analysis, allowing for mother's age, social class, alcohol consumption, and the parity of the baby showed that the mother's smoking habit had a highly significant effect on birthweight (effect $=-253 \mathrm{~g}$, $P<0.001,95 \% \mathrm{CI}=-367$ to $-139 \mathrm{~g})$ and allowing

(C) The Fellowship of Postgraduate Medicine, 1988 
for mother's smoking habit, the father's smoking habit was also significant (effect $=-113 \mathrm{~g}, P=0.030$, $95 \% \mathrm{CI}=-216$ to $-8 \mathrm{~g}$ ). The percentage variance accounted for was only $5 \%$. This effect persisted to 1 month (Table II), when allowing for the factors just given and the babies' age, mother's smoking was associated with a weight difference of $-178 \mathrm{~g}$ $(P<0.01,95 \% \mathrm{CI}=-310$ to $-46 \mathrm{~g})$ and father's with a weight difference of $-112 \mathrm{~g}(P=0.05,95 \%$ $\mathrm{CI}=-224$ to $0 \mathrm{~g}$ ). The interaction between mother's smoking and father's smoking on birthweight was not significant $(P=0.12)$, but was in the direction that the birthweight was further reduced if both parents smoked. There was no significant interaction between mother's smoking and mother's age $(P=0.8)$. Allowing for smoking, there was no effect of alcohol consumption on birthweight. The only significant factor for weight gain was sex $(P=0.026$, girls $2.50 \mathrm{~g} /$ day less than boys, $95 \%$ $\mathbf{C I}=0.28-4.72$ ). Thus babies whose parents smoked grew at the same rate as babies whose parents did not, resulting in the difference in weight persisting at 1 month. The logistic regression showed that mother's smoking was a significant factor in reporting the baby's cough $(P=0.032$, Relative risk $=2.00,95 \% \mathrm{CI}=1.05-3.68)$, but not snuffles/runny nose $(P=0.30$, Relative risk $=1.26$, $95 \% \mathrm{CI}=0.80-2.00)$, having allowed for mother's age, alcohol consumption, parity and type of feeding. There was no additional effect of father's smoking on these symptoms.

\section{Discussion}

We confirm the effect of father's smoking on birthweight; indeed the effect is almost identical to that found by a Danish study. ${ }^{1}$ The major possible source of bias is the under-reporting of cigarette smoking by the mothers, with a possible exaggeration of the amount smoked by other members of the household. However, comparison of the observed prevalence of $47 \%$ and $25 \%$ for men and women respectively with published prevalence figures of $38 \%$ and $33 \%{ }^{6}$ would suggest that any mis-reporting was not large. Further evidence for the mother's truthfulness is that the proportion admitting to drinking during pregnancy is very similar to that of the Danish study. ${ }^{1}$ Gestational age was not routinely recorded in this study and so could not be included in the analysis. A recent large American study ${ }^{2}$ has shown that gestational age is unrelated to passive smoking, however our results are open to the interpretation that passive smoking could be related to prematurity of the babies, rather than growth retardation.

The effect of father's smoking would appear greater when the women admitted to smoking (presumably truthfully), possibly because if the father smokes, the mother is likely to smoke more heavily. ${ }^{1}$ Previous studies demonstrated an effect of both mother's and father's smoking on cough in older children. ${ }^{4,5}$ We have shown that the effect of mother's but not father's smoking extends even to the neonatal period. Perhaps the babies are not directly exposed to their fathers' smoke to any great extent at that age. Thus there is yet further evidence that parents are affecting the health of their newborn children when they smoke, and these facts could perhaps be used to persuade new parents to reduce their smoking.

Table I Mean birthweight (g) by mother's and father's smoking habits

\begin{tabular}{cccccccc}
\hline & & \multicolumn{5}{c}{ Mother } \\
& & Smoker & $(n)$ & Non-smoker & $(n)$ & Total \\
\hline \multirow{2}{*}{ Father } & Smoker & 3183 & $(98)$ & 3431 & $(143)$ & 3330 & $(241)$ \\
& Non-smoker & 3443 & $(30)$ & 3504 & $(247)$ & 3497 & $(277)$ \\
& Total & 3244 & $(128)$ & 3477 & $(390)$ & 3419 & $(518)$ \\
\hline
\end{tabular}

Pooled standard deviation $=501 \mathrm{~g}$.

Table II Mean weight at 1 month (g) by mother's and father's smoking habits

\begin{tabular}{cccccccc}
\hline & & \multicolumn{5}{c}{ Mother } \\
& Smoker & $(n)$ & Non-smoker & $(n)$ & & Total \\
\hline \multirow{2}{*}{ Father } & Smoker & 4012 & $(93)$ & 4238 & $(139)$ & 4147 & $(732)$ \\
& Non-smoker & 4205 & $(31)$ & 4331 & $(240)$ & 4316 & $(271)$ \\
& Total & 4060 & $(124)$ & 4297 & $(379)$ & 4238 & $(503)$ \\
\hline
\end{tabular}

Pooled standard deviation $=628 \mathrm{~g}$. 


\section{Acknowledgements}

We thank the Joint Funding Committee of Southampton and South West Hampshire Health Authority, and the

\section{References}

1. Rubin, D.H., Krasilnikoff, P.A., Leventhal, J.M., Weile, B. \& Beryet, A. Effect of passive smoking on birthweight. Lancet 1986, ii: 415-417.

2. Martin, T.R. \& Bracken, M.B. Association of low birth weight with passive smoke exposure in pregnancy. $\mathrm{Am}$ J Epidemiol 1986, 124: 633-642.

3. Trichopoulos, D. Passive smoking and birthweight. Lancet 1986, ii: 743.
Foundation for the Study of Infant Deaths, who funded this study.

4. Fergusson, D.M., Harwood, L.J., Shannon, F.T. \& Taylor, B. Parental smoking and lower respiratory illness in the first three years of life. $J$ Epidemiol Commun Hlth 1981, 35: 180-184.

5. Charlton, A. Children's coughs related to parental smoking. Br Med J 1984, 288: 1647-1649.

6. Royal College of Physicians. Health or Smoking? Pitman, London, 1983. 